\title{
Perceived Facilitators and Barriers to Exercise Among Older Adults With Mild Cognitive Impairment and Early Dementia
}

\author{
Nicolas Hobson, Sherry L. Dupuis, Lora M. Giangregorio, and Laura E. Middleton
}

\begin{abstract}
Persons with mild cognitive impairment (MCI) and early dementia are often physically inactive, despite associated benefits. This study explored the barriers, facilitators, and preferences for exercise among persons living with MCI/early dementia. The authors conducted 2 focus groups among persons living with MCI/early dementia ( $n=4,6$ participants) and 2 focus groups among care partners ( $n=3,4$ participants). The transcripts were analyzed using thematic analysis, guided by the social-ecological model. Three themes emerged, reinforcing motivation to exercise, managing changes to cognitive and physical health, and variable perceptions of dementia, each with influences from individual, care partner, and community levels. Low intrinsic motivation, poor physical/cognitive health, and stigma restricted the exercise among persons living with $\mathrm{MCI} / \mathrm{early}$ dementia. The care partners motivated their partners and provided company and transportation to exercise. People with MCI/early dementia also indicated poor access to exercise providers and exercise opportunities that met their needs and preferences was a barrier to exercise participation. Knowledge translation research should develop exercise interventions at the individual, social, and community levels.
\end{abstract}

Keywords: behavior change, health promotion, physical activity, socio-ecological model

The worldwide prevalence of dementia was nearly 50 million in 2015 and is projected to reach 131.5 million by 2050 (Prince, Comas-Herrera, Knapp, Guerchet, \& Karagiannidou, 2016). As there is no pharmaceutical treatment to prevent or reverse dementia, alternative approaches to prevent dementia and improve well-being among those with dementia are crucial. People with mild cognitive impairment (MCI) have cognitive impairment and a high risk of dementia, but still retain intact activities of daily living (Petersen et al., 2014; Strout \& Howard, 2012). MCI could be a pragmatic stage for intervention, where individuals have a clear diagnosis and high risk for dementia, but retain much of their abilities.

Exercise is one potential strategy to improve wellness among older adults with MCI or dementia. Exercise improves functional abilities, physical function, and possibly cognitive function among older adults with MCI or dementia (Forbes, Blake, Thiessen, \& Forbes, 2015; Ginis et al., 2017; Öhman, Savikko, Strandberg, \& Pitkälä, 2014). Although the benefits to physical and cognitive function are often emphasized, exercise has broader benefits to well-being and social inclusion for persons living with dementia (Cedervall, Torres, \& Aberg, 2015; Malthouse \& Fox, 2015). Longtime exercisers, in particular, see exercise as a personally meaningful activity; accessing exercise is one way to exert choice and control in their life and to maintain personal identity (Phinney, Chaudry, \& O'Connor, 2007; Van Alphen, Hortobágyi, \& van Heuvelen, 2016).

Despite the broad benefits of exercise, persons living with MCI or dementia remain less physically active than older adults with healthy cognition (McGuire, 2014), who are themselves often inactive. Despite the low exercise participation among persons living with MCI or dementia, relatively few studies have investigated the barriers to exercise among these groups (Cedervall \& Aberg, 2010;

Hobson, Giangregorio, and Middleton are with the Department of Kinesiology, University of Waterloo, Waterloo, Canada. Dupuis is with the Department of Recreation and Leisure, University of Waterloo, Waterloo, Canada. Giangregorio and Middleton are also with the Research Institute for Aging, Waterloo, Canada. Middleton (laura.middleton@uwaterloo.ca) is corresponding author.
Cedervall et al., 2015; Chong et al., 2014; Dal Bello-Haas, Connell, Morgan, \& Crossley, 2014; Malthouse \& Fox, 2015; Suttanon, Hill, Said, Byrne, \& Dodd, 2012; Tak, van Uffelen, Paw, van Mechelen, \& Hopman-Rock, 2012; Yu \& Kolanowski, 2009; Yu, Savik, Wyman, \& Bronas, 2011). Studies that do exist have focused on the individual-level factors and have confirmed that physical and cognitive deficits clearly limit exercise among people with MCI or dementia, and that care partners and exercise providers are important facilitators of exercise. However, these studies failed to deeply probe the factors related to the social and physical environments. One study indicated that a poor understanding of dementia can limit exercise participation, but admitted that their question guide did not explicitly probe social or environmental factors (Malthouse \& Fox, 2015). Relational social models of disability suggest that disability is caused by an interaction between the individual and the environment. (Kattari, Lavery, \& Hasche, 2017; Thomas, 2004). In considering the factors that influence exercise among persons living with MCI or dementia, it is useful to direct attention to the factors at multiple levels (McLeroy, Bibeau, Steckler, \& Glanz, 1988).

The factors that affect participation in exercise can be classified within the conceptual framework of the social-ecological model (SEM). The SEM posits that behavior is affected by the complex and dynamic interaction of factors on multiple levels (McLeroy et al., 1988). Framing issues within the SEM can help people to understand how macrolevel systems (e.g., the social and physical environment) influence microlevel (e.g., individual) decisions, behaviors, and attitudes (Henderson \& Baffour, 2015). Furthermore, purposeful probing and identification of barriers within the social and physical environment may provide actionable steps to inform behavior-change strategies at these levels. The overarching aim of this study was to understand the barriers, facilitators, and preferences for exercise among persons living with MCI or early dementia from the perspectives of persons living with $\mathrm{MCI}$ or early dementia and their care partners using the SEM as a guiding framework. Care partners, in this context, are a strong interpersonal determinant of exercise among persons living with MCI or early 
dementia. In this study, we have narrowed our focus to people living with MCI or early dementia, as opposed to more progressed stages, as it is reasonable to expect that many people with MCI or early dementia could feasibly participate in community-dwelling exercise, given minor support from their social network and community.

\section{Methods}

Participants were purposively recruited from the Kitchener-Waterloo area (Ontario, Canada). Although our recruitment was geographically restricted, we purposively recruited persons living with MCI or early dementia (from local Alzheimer Society groups, dementia-advocacy groups, dementia-related studies, and dementiarelated community events) who were interested in being active, but may be currently active or inactive, and care partners to persons with MCI or dementia who fit this description. In this way, they could meaningfully comment on the barriers they had experienced and could reflect on the supports that helped or could have helped them be active. The eligibility criteria were left intentionally broad to provide the opportunity to elicit diverse personal experiences. To be eligible for the study, persons living with MCI or early dementia had to (a) live in the community, (b) have a self-reported clinical diagnosis of either MCI or early dementia, and (c) be above the age of 55 years. Similarly, care partners had to (a) be the primary care partner for someone diagnosed with MCI or early dementia, (b) live in the community, and (c) not have a current clinical diagnosis of MCI or dementia. However, the participants did not need to participate in dyads. That is, the care partner of a participant living with MCI or early dementia did not need to participate in the study, nor the reverse. Furthermore, if both partners were in the study, they did not necessarily participate on the same day.

In total, 17 participants were recruited to the study, including 10 persons living with MCI or early dementia (five with MCI and five with dementia) and seven care partners. This study received ethics clearance from the University of Waterloo Office of Research Ethics (ORE reference \# 21362). All of the participants provided written informed consent. Although we had the option for care partners to provide consent if the person with MCI or early dementia did not have a sufficient capacity to understand the study procedures, this option was not used by any participant.

\section{Procedure}

Each participant attended one study session at the University of Waterloo's Centre for Community, Clinical and Applied Research Excellence building between January 2017 and March 2017. The participants (with their partner, if present) were brought to a quiet room where they were free to ask any questions and sign the consent forms. The participants then completed a questionnaire that was administered in a structured interview format, which engaged the care dyad together if both were present. The questionnaire gathered basic information about the participants, their health, and their exercise participation. Following the questionnaires, the participants congregated in one of two groups for a focus group discussion, where the persons living with MCI or early dementia and care partners participated in separate focus groups.

\section{Questionnaires}

The participants reported demographic characteristics including age, gender, marital status, and the highest level of education achieved. The participants also reported details about their living arrangement, including an urban or rural setting, the persons they lived with, and the type of residence (private home/apartment, seniors' residence, or other). The participants also reported their current health conditions, falls in the last 2 months, and use of mobility aids. Finally, the participants reported their exercise habits and intentions to exercise over the last 6 months using the Stages of Change Questionnaire (Chong et al., 2014; Marcus, Selby, Niaura, \& Rossi, 1992).

\section{Focus Groups}

Focus groups were used to explore barriers to exercise/physical activity, which may elicit a greater breadth of responses and discussion than interviews and, thus, broaden the scope of subsequent analyses and conclusions (Morgan, 1996). The persons living with dementia and care partners participated in separate but concurrent focus groups, creating a relatively homogenous group of participants to avoid major power imbalances and allow the participants to share freely in an open and comfortable environment. Although some recommend a focus group size between six and 12 participants to promote diversity but maintain cohesion (Krueger, 2000; Onwuegbuzie, Dickinson, Leech, \& Zoran, 2009), participants with MCI or early dementia may be more comfortable sharing their thoughts in a smaller group size (Sutcliffe, Roe, Jasper, Jolley, \& Challis, 2015). Thus, a total of four focus groups, two focus groups with persons living with MCI or early dementia (focus group 1: four persons, focus group 2: six persons) and two focus groups with care partners (focus group 1: three persons, focus group 2: four persons), were conducted.

A moderator (persons living with dementia: N.H.; care partners: M.V.) and an assistant moderator (persons living with dementia: C.M.; care partner: M.B.) facilitated each focus group. To start the focus group, the moderator and assistant moderators introduced themselves, briefly explained the study rationale, purpose, procedures, role of the moderator and assistant moderator, and how the anonymity of responses would be maintained in transcripts through coded identifiers. They further explained a set of ground rules for the discussion. The moderators then opened the focus group with an introductory question that each participant answered. The remainder of the focus group discussion was facilitated in a semistructured manner, referring to the focus group discussion guide as necessary. The focus group discussion guide included open-ended questions and prompts specific to each participant group (Appendix A). The focus of the prompts was the exercise experiences, barriers, and facilitators of persons living with MCI or early dementia, though the exercise habits of care partners were also discussed. The formulation of the focus group discussion guide was informed by the SEM to probe the individual, sociocultural, and environmental influences on exercise.

Both the moderator and assistant moderator took field notes during the focus group discussion for the purpose of debriefing and providing clarity. The assistant moderator audio-recorded the session. Focus group discussions lasted between 60 and $90 \mathrm{~min}$ (mean of $79 \mathrm{~min}$ [SD=7 min]) with a brief break midway. The goal was to promote discussion until natural expiration. At the conclusion of each focus group, the assistant moderator provided a brief summary of the main points raised during the discussion and gave the participants the opportunity to verify or clarify these points. 


\section{Data Analysis}

Audio recordings of the focus groups were transcribed verbatim. Repeated readings of the transcribed focus group data by the lead author (N. Hobson) were then conducted to develop a thorough understanding of the data. The focus group transcripts were analyzed using general inductive thematic analysis with a focus on constant comparisons (Braun \& Clarke, 2006). Initial codes were generated by N. Hobson through line-by-line coding with the goal to describe the key concepts evident in each particular line. In particular, words, phrases, and sentences that appeared relevant to the research objectives were highlighted and manually coded. To ensure coding consistency, independent parallel coding was carried out by two secondary coders. The codes developed by the initial and secondary coders were then discussed to compare the extent of overlap that existed. Since coding had a high degree of conceptual agreement, coding schemes were merged. The codes were sorted into potential themes and categories based on the similarity of content and meaning. The categories and assignment of text to categories were scrutinized for consistency and clarity by secondary coders to ensure that the interpretations were sound. Finally, developing themes and subthemes were named, combined, and linked under core concepts. Guided by the SEM framework, a cross-level framework of subthemes was created. At all stages of the analysis process, memo writing through code notes and field notes was made in a reflexive journal and utilized to provide further contextual insights to illustrate the development of core ideas and themes. This also allowed for further scrutiny over the decisions that were made during analysis.

\section{Trustworthiness}

Careful processes were used to ensure trustworthiness of the results by procedures that addressed credibility, transferability, dependability, and confirmability (Onwuegbuzie \& Leech, 2007; Shenton, 2004). Member checks were also conducted by the assistant moderator at the end of each focus group so that the participants could verify the interpretations of the moderating team. The credibility of the findings was also enhanced through frequent debriefing sessions held with peers, coinvestigators, and advisors to allow the opportunity for peer scrutiny. To improve transferability, a detailed description of the study's setting, participant population, and participant selection is provided in the Methods Section. To support dependability, an audit trail was established by maintaining extensive, detailed descriptions of reflexive thoughts, the study methodology, data collection, analysis decisions, and processes. Confirmability was enhanced through investigator triangulation whereby a secondary moderating team (moderator and assistant moderator) acted to balance the predispositions of the researcher by offering a unique set of similar yet distinct perspectives. The preservation of audio recordings, field notes, and debriefing notes further contributed to the audit trail and strengthened the confirmability of this study's findings.

\section{Results}

The participants with MCI or early dementia had an average age of $75.3(S D=6.5)$ years and an average of $14.2(S D=4.0)$ years of education; three were women and seven were men. The care partners had an average age of $74.4(S D=7.0)$ years and an average of $14.1(S D=4.8)$ years of education; six were women and one was a man. Additional participant characteristics by participant type are presented in Table 1.

\section{Table 1 Participant Characteristics by Participant Group}

\begin{tabular}{lcc}
\hline Characteristic & $\begin{array}{c}\text { Person with } \\
\text { MCI or early } \\
\text { dementia }(\boldsymbol{n}=\mathbf{1 0})\end{array}$ & $\begin{array}{c}\text { Care } \\
\text { partner } \\
(\boldsymbol{n}=\mathbf{7})\end{array}$ \\
\hline Age (years) & $75.3(6.5)$ & $74.4(7.0)$ \\
Gender (\%female) & 3 & 6 \\
Body mass index $\left(\mathrm{kg} / \mathrm{m}^{2}\right)$ & $26.5(5.0)$ & $25.6(3.1)$ \\
Education (years) & $14.2(4.0)$ & $14.1(4.8)$ \\
Marital status (\%married) & 8 & 7 \\
Diagnosis (\%MCI) & 5 & - \\
Time of diagnosis, <5 years (\%) & 9 & - \\
Residence & & 4 \\
$\quad$ Urban (\%yes) & 7 & - \\
$\quad$ Alone (\%yes) & 1 & 1 \\
Mobility aid (\%yes) & 1 & 1 \\
Falls in past 2 months (\%yes) & 2 & 1 \\
Comorbid health conditions (\%no) & 2 & 4 \\
Current exerciser (\%yes) & 8 & $60(0-315)$ \\
Exercise volume (min/week) & $127.5(0-600)$ & \\
\hline
\end{tabular}

Note. Data are presented as mean $(S D), n$, or median (minimum-maximum). Median (minimum-maximum) is used only for exercise volume. $\mathrm{MCI}=$ mild cognitive impairment.

Themes and subthemes were developed inductively based on the focus group discussions. Thematic analysis revealed a complex and dynamic interplay between factors influencing exercise behavior among persons living with MCI or early dementia across levels of the SEM. The factors influencing the themes emerged across three levels: (a) the individual, (b) the care partner (and social network), and (c) the community (leaders, services, and infrastructure). Table 2 summarizes the themes and the subthemes that contributed to each theme by level. Participant quotes are labeled by participant type (PWMD [person with MCI or early dementia] or CP [care partner]), the day of the focus group (1 or 2), and a number referring to individual within that focus group (but a number).

\section{Theme 1: Reinforcing Motivation to Exercise}

Individual level: Diverse motivators, but restricted by day-to-day motivation. The persons living with MCI or early dementia expressed several motivations to exercise, including their diagnosis as a motivator, as well as their belief in the benefits of exercise. However, they also experienced difficulties with intrinsic motivation day to day.

The diagnosis of MCI or early dementia itself was a motivation to exercise more. When asked how their diagnoses influenced their willingness or opportunities to exercise, one person living with MCI or early dementia responded, "Mine have increased 'cause I didn't exercise before [the diagnosis] . . I I do more exercise now than I ever did" (PWMD1-2). The persons living with MCI or early dementia did not believe that their condition negatively affected exercise opportunities; rather, they were motivated by the possibility of improving their physical and mental functioning.

The persons living with MCI or early dementia also experienced benefits to their own physical and mental health, which prompted them to do more exercise. A person living with MCI or 
Table 2 Overview of Emergent Themes and Subthemes by Primary Level of Influence

\begin{tabular}{|c|c|c|c|}
\hline \multirow[b]{2}{*}{ Theme } & Intrapersonal & $\begin{array}{l}\text { Care partner and social } \\
\text { network }\end{array}$ & Community \\
\hline & \multicolumn{3}{|c|}{ Subthemes } \\
\hline $\begin{array}{l}\text { Reinforcing } \\
\text { motivation to } \\
\text { exercise }\end{array}$ & $\begin{array}{l}\text { - Diagnosis as motivation } \\
\text { - Health and personal benefits } \\
\text { - Low intrinsic motivation day to day }\end{array}$ & $\begin{array}{l}\text { - Encouragement from care part- } \\
\text { ners } \\
\text { - Exercise as a social activity } \\
\text { - Social engagement with others } \\
\text { whom they could identify with, } \\
\text { which could be by cognitive/carer } \\
\text { status, age, and gender }\end{array}$ & $\begin{array}{l}\text { - Encouragement by exercise } \\
\text { providers } \\
\text { - Advice of family physician } \\
\text { - Access to preferred forms of } \\
\text { exercise through infrastructure } \\
\text { and programming } \\
\text { - Access to enjoyable group } \\
\text { exercise }\end{array}$ \\
\hline $\begin{array}{l}\text { Managing } \\
\text { changes to cog- } \\
\text { nitive and phys- } \\
\text { ical health }\end{array}$ & $\begin{array}{l}\text { - Changes in physical health restrict } \\
\text { exercise (e.g., mobility, arthritis) } \\
\text { - Memory and confusion restrict exercise } \\
\text { - Day-to-day variability in cognitive/ } \\
\text { physical health }\end{array}$ & $\begin{array}{l}\text { - Reliance on care partners to } \\
\text { accompany and facilitate to } \\
\text { exercise } \\
\text { - Current or future reliance on } \\
\text { care partners for transportation } \\
\text { - Restrictions due to care part- } \\
\text { ner's health } \\
\text { - Restrictions due to care part- } \\
\text { ner's concerns for safety }\end{array}$ & $\begin{array}{l}\text { - Infrastructure that accommo- } \\
\text { dates needs } \\
\text { - Exercise programs and provi- } \\
\text { ders that accommodate their } \\
\text { needs } \\
\text { - Accessible public transportation }\end{array}$ \\
\hline $\begin{array}{l}\text { Variable percep- } \\
\text { tions of } \\
\text { dementia }\end{array}$ & $\begin{array}{l}\text { - Retained capacity } \\
\text { - Optimism }\end{array}$ & $\begin{array}{l}\text { - Perception of loss } \\
\text { - Proactive in management }\end{array}$ & - Stigma \\
\hline
\end{tabular}

early dementia noted, "Especially if you can notice the difference [of exercise on health], I mean that makes you motivated to keep doing it" (PWMD2-1) and "If you're out walking or doing something . . . you just feel uplifted. From my point of view, [exercise] has really helped me" (PWMD1-3). Similarly, others responded that exercise improved their mental function; one stated, "One leads to the other [exercising leading to better thinking]" (PWMD4-2).

Despite the motivation associated with their diagnosis, both persons living with MCI or early dementia and their care partners reported that lack of intrinsic motivation among persons living with MCI or early dementia was a major barrier to exercise. They believed they should exercise more, but lacked the drive to do it: "I get in the way [of exercising] . . . I don't know, sometimes I think I'm just too lazy" (PWMD2-3). The care partners believed that the lack of intrinsic motivation exhibited by their partner with MCI or early dementia was likely part of their nature and not due to dementia. They indicated that their partners with MCI or early dementia also had difficulty overcoming obstacles to exercise in the past: "If he didn't have dementia, he'd probably be the same ... he won't go swimming, he won't go to yoga" (CP1-1).

Care partner and social networks: Profound influences among social connections. The persons living with MCI or early dementia indicated that the encouragement and support from others was a major motivator to exercise and a determinant of their exercise behavior-most prominently, but not exclusively, coming from their care partner. The care partner was a major influence on their exercise behavior, usually to promote exercise: "My wife, uh, has always been pretty keen on doing exercises, so that makes it easier for her and for me" (PWMD1-1). The care partners thought that their role in motivating exercise and other activities increased after the diagnosis of MCI or early dementia of their partners. They were driven to get their partner with MCI or early dementia to exercise because they believed that exercise was beneficial: "We're going to keep exercising, and we're going to keep whosever [is] in our lives [exercising] . . . I don't know how you would be able to motivate [someone with dementia] if they had caregivers who didn't have that lifelong connection with exercise" (CP2-4).

Beyond encouragement, social engagement at programs also motivated the persons living with MCI or early dementia to exercise. Having a companion to exercise with was unanimously agreed to be a strong motivator for exercise among the persons living with MCI or early dementia: "It's a lot easier [to exercise] if you have somebody to do things [with]. Helps to have somebody with you. The other person . . . is a good incentive [to exercise]" (PWMD2-2). This companion was typically, but not always, their care partner.

Persons living with MCI or early dementia viewed exercise as a social activity and appreciated that they received "double benefits, physically and socially" (PWMD3-2). Some participants indicated that exercise and social engagement were inseparable from one another and amplified the benefits: "I think the two [exercise and social activity] are [together] and [are] an opportunity to socialize and improve your physical [health]. I also found that the social dimension of exercising kind of brightens the day too" (PWMD1-1). Social engagement also incited an ongoing adherence to exercise in order to maintain relationships: "You meet some nice persons too, you know, when you exercise. [Socializing] adds incentive to, uh, going back" (PWMD2-2).

Social engagement during exercise programs was particularly motivating when the persons living with MCI or early dementia participated with others whom they could identify with, which could be by cognitive/caring status, gender, and/or age. Such engagement induced feelings of comfort and enjoyment, and a sense of self-worth. The persons living with MCI or early dementia emphasized how much they enjoyed being with others who understand and accept them and the condition they have: "To be able to be with others and speak a little bit and not have ... [to] 
speak like you're speaking to someone who knows nothing about what's going on in your life ... it's great" (PWMD2-3).

It was clear that the persons living with MCI or early dementia still identified as much by their gender and age as by their diagnosis. The characteristics of group exercise participants influenced their enjoyment and, as a result, their adherence to exercise programs: "I did, uh, a program at one of the gyms a few years ago that was sort of geared to a younger crowd, and I didn't particularly enjoy [it]" (PWMD1-1). Another added, "He [partner with MCI or early dementia] went back to do the exercises to keep it up. He'd be right with the women ... well, he felt so out of place, not enjoying it" (CP1-1).

Community: Programs, providers, and environment can improve motivation. Encouragement from a range of community leaders also motivated the persons living with MCI or early dementia to exercise. The persons living with MCI or early dementia believed that having a knowledgeable, understanding, and friendly exercise provider, and a welcoming environment were important to motivating and enabling exercise. They indicated that they enjoyed exercise more when they had a friendly exercise provider who was encouraging: "Having a good teacher I think is vital . . . very encouraging" (PWMD2-5). Persons living with MCI or early dementia expressed a specific desire for "more trained persons" to better understand the exercise needs of persons living with dementia: "[They] need to be paying attention to what [persons living with dementia are] doing and give them some encouragement and welcome them when they come in" (PWMD2-3).

The persons living with MCI or early dementia also indicated that recommendations from their family physician motivated them to exercise, though it was unclear whether exercise was recommended for general health or specifically for their condition. In some cases, the advice from a physician induced a sense of urgency and fear, inciting the participants to initiate and maintain their exercise habits: "He [the doctor] says, 'You know you got a serious problem, although you're not sick, but that weight's gonna kill you.' He motivates me. He's the one that said, 'You're going to die", (PWMD1-2).

Access to preferred exercise opportunities within the community improved individual motivation to exercise. Walking outdoors was frequently identified as a preferred mode of exercise, which could be facilitated or restricted by the physical environment. Pleasant locations to walk (e.g., parks and forests) motivated walking for exercise among persons living with MCI or early dementia. However, weather could reduce the motivation to walk outdoors: "If it's too hot or too cold or wet, I just wouldn't go out [for a walk]" (PWMD1-4).

The participants, particularly the care partners, suggested that access to group exercise programs that aligned with their preference would entice them to exercise, but these were seldom available in the community. Access to dementia-specific programs could motivate exercise by providing an opportunity to socialize with others who understand them, by tailoring exercises to physical and mental needs and by providing fun and enjoyable environments. They suggested that motivation to undertake an exercise program would be enhanced with increased availability: "The problem that we've had is we've never found the kind of exercise program [that is appropriate and that they like]. We'd love to do it. I think there should be more group programs [for persons living with MCI or early dementia]" (CP2-4). Access to suitable programs was not restricted to the dementia-only groups. Motivation to exercise could also be enhanced with better availability of social (group) exercise for older adults, where persons living with MCI or early dementia and care partners could be included. In particular, if there were opportunities to engage with individuals they identified closely with: "It would become fun [attending exercise program with older adults who are similar], something you don't want [to] miss out on, meet some nice persons and have a good time" (CP2-3).

\section{Theme 2: Managing Changes to Cognitive and Physical Health}

Individual level: Physical and mental limitations make participation more difficult. Changes in physical and mental health influenced the capacity and willingness of the persons living with MCI or early dementia to exercise. Physical impairments, including mobility restrictions, pain and arthritis, visual impairment, and other physical changes, limited the ability of the persons living with MCI or early dementia to exercise, particularly walking for exercise. For example, one person living with MCI or early dementia indicated, "I would love dearly to get down there [to the forest], but my hips won't let me right now . . . my arthritis is worse, pain and the movement I could do . . . I can't keep it up" (PWMD2-2). Exercise was also limited by fatigue among the persons living with MCI or early dementia: "It's just that . . . I don't have the energy; I'm tired, and so I just don't do it [exercise]" (PWMD2-1).

Memory problems and confusion among persons living with MCI or early dementia could also interfere with exercise, particularly exercise at home: "Remembering is a really big problem. Did I do that one [exercise]?" (PWMD2-6). Exercise was said to be particularly limited when the person living with dementia was "having a bad day." The care partners also indicated that low mood and depression could negatively influence the exercise levels of the person living with MCI or early dementia, perhaps more so than cognitive changes.

Care partner: Key to overcoming cognitive barriers, cognitive changes increase reliance. Cognitive challenges associated with MCI or early dementia led to an increasing reliance on their care partners as a companion and exercise chaperone: "Cognitively, he just seems to struggle. He can't remember . . . it almost seems as if he can't retain the information . . . and so I have to be there with him to do it [exercise]" (CP2-3). This included navigating local areas during walking exercise: "I just uh, can't do it [walk alone] anymore .. . I usually need my wife to go with me 'cause sometimes I can't remember where to go" (PWMD1-1).

The care partner's provision of transportation was also critical to facilitating exercise for the persons living with MCI or early dementia, who often could not drive themselves or knew that they might lose their license in the future. The persons living with MCI or early dementia were clear that access to exercise opportunities would be extremely limited if not for their care partner driving: "Fortunately, my wife is very supportive, and, um, enjoys the exercises too [referring to driving him]" (PWMD1-1). Another added, "Transportation in some ways is an issue for me if my wife isn't able to drive [referring to driving to exercise programming opportunities]" (PWMD1-2).

Although the care partners usually encouraged and facilitated exercise among the persons living with MCI or early dementia, this was not always the case. The care partner's facilitation of exercise was sometimes hampered by their own health issues. One care partner said, "[We] used to walk a lot . . . but for the last year or so, this knee has completely stopped all that" (CP1-1). Furthermore, concerns about safety due to physical and mental health changes sometimes made the care partners hesitant to facilitate exercise for 
persons living with MCI or early dementia: "I used to do it [cross country ski], but, then again, my wife has to drive me and she doesn't cross country ski and, well, she's not so keen on me going by myself in case I fall" (PWMD1-1).

Community: Accessible planning facilitates participation and inclusion. Safe and accessible community infrastructure and exercise programming were thought to facilitate exercise for persons living with dementia or restrict exercise if these were lacking. Many of the persons living with MCI or early dementia felt that there were exercise opportunities for them, particularly for walking, which was a preferred activity. However, high cycling or pedestrian traffic could hinder the use of trails and sidewalks. Poor sidewalk conditions could make them unsafe for walking, especially among individuals with physical limitations: "Some of the sidewalks they've been working on have been just too dangerous to walk on . . . sometimes they're not level. The surface you're walking on is important!" (PWMD1-3). Furthermore, community infrastructure did little to facilitate exercise if it did not align with the individuals' needs: "I'm limited on the machines I can use too, locked in on the bike with my shoes or I'm on the rowing machine, so I can't; it's 'cause I have a little bit of balance problem" (PWMD1-4).

The participants also described program characteristics that would facilitate their participation in organized group exercise: "Having somebody working with you, if it's not hard to get there, and if it's not too expensive ... I think I would [participate in that exercise program]" (PWMD1-3).

The care partners were aware of dementia-specific exercise programs in the community. Some of the persons living with MCI or early dementia explained that there were barriers even to accessing these dementia-specific programs. Most of the programs required a care partner to be present, which was not possible for all of the persons living with MCI or early dementia: "As with all these programs [for persons living with dementia], you have to have your care partner with you, and I knew I wasn't going to get that, so I didn't even look at it" (PWMD2-2). This was viewed as a problem in program design rather than one of care partner availability.

Access to exercise opportunities that accommodate the needs of individuals with MCI or early dementia was further restricted by transportation, the influence of which could be affected by the care partner (as previously introduced), but also by community planning. The persons living with MCI or early dementia often could not drive themselves and sometimes could not rely on care partners. At the time of this study, the persons living with MCI or early dementia did not qualify for community-assistive transport for persons with disabilities. This community service that was meant to support individuals with a disability specifically excluded persons living with MCI or early dementia. The care partners described an active initiative to challenge these rules: "There's a drive on to have persons with dementia classified as disabled so they can use disabled transport; right now, dementia does not count as a disability" (CP1-3).

\section{Theme 3: Variable Perceptions of Dementia}

Individual: Optimism despite disease. The perceptions that the persons living with MCI or early dementia had about dementia were rooted in optimism for the future and suggested that having the disease was manageable: "I would like to let persons know that if you do have it [dementia], it's not the end of the world" (PWMD2-4). They felt relatively unaffected by the disease and suggested that being diagnosed with MCI or early dementia had little impact on their life at this stage: "It hasn't affected me in the least ... I mean, the fact that I have [dementia]. Not worried about it and, so, I just carry on as usual, as if I never had it" (PWMD2-4). This optimism regarding their condition and their future made them feel relatively unimpeded for exercise. Another expressed that, even though he or she had recently been diagnosed, they was "gonna keep doing what I'm doing and do more [walking for exercise]" (PWMD2-3).

Care partners: $\boldsymbol{A}$ dissonance of loss and action. The care partners' view of dementia was in strong contrast to that of the persons living with MCI or early dementia. The care partners described dementia as an experience of loss: "Dementia's called the long goodbye because the person you knew is going away and somebody else is coming" (CP2-3). The care partners confirmed that they felt more affected by their partners' diagnoses than their partner living with MCI or early dementia did: "I think it bothered me more when I heard [the diagnosis]; he's [spouse with MCI or early dementia] been a real trooper through it all" (CP2-4). Despite the profound impact of their partners' diagnoses of MCI or early dementia, the care partners indicated that it is necessary to be proactive when confronted with the observable and significant symptoms of MCI or early dementia. One said, "It's manageable, but I also see [caring for my partner with dementia] as a non-stop battle . . . if you back off with your own constant vigilance, it just then comes back with a vengeance" (CP1-2). Having this perspective encouraged the care partners to facilitate exercise for their partners with MCI or early dementia. The care partners' perspectives were often informed by prior experiences with family members who had AD: "I would like persons to watch out for its [dementia's] early stages and not leave it out till it's too late" (CP1-3).

Community level: Perceived societal stigma. The persons living with MCI or early dementia were concerned about societal-level stigmatization and medicalization of dementia, which ultimately had a negative influence on exercise participation. They felt that persons perceived them as being a product of the disease they had, a "feeling that in this stage, I'm identified by [the] disease I have. So that's something I've wrestled with, and I guess the hesitation I have sometimes in telling others what my diagnosis is; how are they going to interpret that?" (PWMD1-1). Contrary to this, some persons with MCI or early dementia had positive experiences of understanding and patience by those who were aware of their dementia diagnosis. One person expressed their pleasure when interacting with others who were compassionate and were able to "throw in their awareness and understanding of what's involved [regarding dementia]" (PWMD3-1).

\section{Discussion}

This study explored the barriers, facilitators, and preferences for exercise among community-dwelling older adults diagnosed with MCI or early dementia from the perspective of persons living with MCI or early dementia and their care partners. Several barriers identified by our participants were similar to those identified by older adults, more broadly. Optimistically, the changes in the social and physical environment to overcome these shared barriers (e.g., physical challenges, low motivation) would promote both dementia- and age-friendly initiatives. Still, there are several unique barriers and facilitators to exercise among persons living with dementia. These include the severity of cognitive deficits, reliance on their care partner, and poor understanding of and stigma against dementia. Not previously acknowledged in literature, our 
participants indicated that a lack of accessible exercise opportunities specifically targeted to or inclusive of older adults with dementia was a barrier to exercise. This may be reflective of a societal-level stigma, where there is a failure to acknowledge the retained capacity among this population. Our findings highlight how changes in the community might be employed to help persons living with MCI or early dementia overcome individual-level barriers to exercise, an area to be explored in future studies.

Many barriers experienced by persons living with MCI or early dementia are not unique, including physical challenges and low motivation to exercise. Reduced mobility and pain are common barriers to exercise among older adults (Franco et al., 2014; Schutzer \& Graves, 2004) and have been reported here and in prior studies of persons living with MCI and Alzheimer's disease (Cedervall \& Aberg, 2010; Chong et al., 2014; Malthouse \& Fox, 2015). A one-size-fits-all strategy to deliver exercise cannot address the diverse individual needs of older adults (Schutzer \& Graves, 2004). Facilities and programs that can accommodate the diverse physical needs of older adults are critical to both age- and dementiafriendly initiatives (Costello, Kafchinski, Vrazel, \& Sullivan, 2011; O'Brien, Dodd, \& Bilney, 2008; Suttanon et al., 2012; World Health Organization, 2007).

Low intrinsic motivation is a major barrier to exercise across all ages and abilities (Franco et al., 2014; Martins, Marques, Sarmento, \& Carreiro da Costa, 2015). It is reasonable to think that apathy, a common symptom of dementia (David et al., 2012), could accentuate low motivation. Nonetheless, the care partners in this study reported that low motivation to exercise among the persons living with MCI or early dementia generally preceded the diagnosis. On the contrary, the diagnosis of MCI or early dementia itself was considered a motivator to exercise. Some persons reported that they exercised more after their diagnosis than before. Persons living with MCI or early dementia report that physical activity can contribute to well-being and maintained identity after a diagnosis of MCI or early dementia (Cedervall et al., 2015; Menne, Kinney, \& Morhardt, 2002).

While changes in physical health are common with aging, persons living with MCI or early dementia experience more severe cognitive deficits than is typical. The cognitive deficits mean that they are reliant on others to facilitate exercise. In this study, the care partners were the most common significant influence on the exercise levels of their partner with MCI or early dementia, confirming several prior studies (Malthouse \& Fox, 2015; Suttanon et al., 2012; Van Alphen et al., 2016, Yu et al., 2011). The care partners provided transportation and encouragement, and accompanied their partners living with MCI or early dementia for exercise. In this study, several care partners expressed a strong belief in the benefits of exercise, which drove them to encourage, support, and facilitate exercise for their partners. In contrast, our participants indicated that poor health of the care partner or lack of care partner support for exercise was a significant barrier to exercise among persons living with MCI or early dementia. For these persons living with MCI or early dementia, broader accessibility initiatives in the community are particularly important.

The participants believed that access to exercise that is enjoyable and inclusive promoted motivation and exercise participation among the persons living with MCI or early dementia. By adapting exercise and program delivery, exercise providers could enable persons living with MCI to overcome physical and cognitive challenges (Black \& Rush, 2002). Unfortunately, exercise providers often have little knowledge and training regarding dementia (Bechard, Mitchell, Regan, Bergelt, \& Middleton, 2018). Improved training of exercise providers regarding MCI or early dementia would promote dementia-friendly exercise opportunities.

The participants reported that social engagement with others of a similar cognitive/carer status was motivational for exercise, confirming prior research (Dal Bello-Haas et al., 2014; Yu et al., 2011). Dementia-specific programs are available (e.g., day programs). However, these programs often lack the capacity to include all persons living with MCI or early dementia and are frequently too low in intensity to promote fitness among persons who still have good physical function. Broader access to exercise programs supports inclusion and increases the number and variety of programs available. Furthermore, it allows persons living with MCI or early dementia to continue to engage with the broader community. One important point highlighted by our participants, not recognized in prior literature, was that they continued to identify by age and gender. As such, interaction with such peers was also an important motivator for exercise.

The perception of dementia more broadly in society has a profound influence on behavior (including exercise) of persons living with dementia. In this study, our participants highlighted differences in how dementia was perceived between the persons living with MCI or early dementia, the care partner, and broader society. Unique among studies of barriers to exercise, our participants living with MCI or early dementia perceived little change in their abilities (particularly in their ability to engage in exercise) and often approached the future with optimism. This may be related to increased dementia advocacy efforts, which aim to portray dementia as a disability that that does not preclude ongoing participation and activity (Alzheimer's Disease International and Dementia Alliance International, 2016; Crowther, 2016; United Nations, 2008). In contrast, care partners viewed dementia as a progressive loss.

In this and other studies, persons with early stage dementia have indicated they are aware of a societal stigma against dementia, causing worry about how others may respond to their diagnoses (Reed \& Bluethmann, 2008). Fear of public embarrassment discourages persons living with MCI or early dementia from participation in activities, including exercise (Ashworth, 2017; Cedervall \& Arberg, 2010; Husband, 2000). It is likely that improved public education and awareness could reduce stigma (among the public) and fear of stigma (among persons living with MCI or early dementia and care partners) and thereby promote the social participation of persons living with MCI or early dementia in activities such as exercise.

Prior studies of barriers to exercise did not carefully probe societal-level influences. Here, our participants indicated that perceived societal stigma of dementia discouraged participation in exercise. Moreover, it is likely that the societal-level stigma against dementia contributes to the failure to create accessible opportunities and accommodates persons living with dementia in exercise programs and other community services. Persons living with MCI or early dementia are often discounted in social contexts and perceived as lacking the capacity to participate (Alzheimer's Europe, 2013). Even accessible services, such as adaptive transportation, do not always include persons living with MCI or early dementia. Transportation was a significant barrier to exercise noted here and in prior studies by older adults with and without cognitive impairment (Boehm et al., 2013; Yu et al., 2011). At the time of this study, local accessible transportation specifically excluded persons living with dementia. Around the time of the focus groups, however, independent advocacy efforts have led to the inclusion of dementia as a disability with full rights under the United Nations Convention on the Rights of Persons with Disabilities (Alzheimer's Disease International and Dementia Alliance International, 2016; 
Crowther, 2016; United Nations, 2008). As a result, government infrastructure and programs are increasingly being adapted to accommodate the needs of persons living with MCI or early dementia. Some countries are more advanced in this respect; for example, recent guidance from the United Kingdom provides recommendations for dementia-friendly sport and physical activity (Alzheimer's Society, 2019), though recommendations are based on general dementia-friendly research and not exercise-specific inquiries. Broad inclusion of persons living with MCI or dementia remains a work in progress, but one that could enable the physical and social participation of persons living with MCI or early dementia in the community.

This study was the first to explicitly probe the factors that influence exercise among older adults with MCI or early dementia using a socio-ecological framework. As such, our results provide insights on the interactive influences on exercise across the individual, social, and community levels. However, our sample was drawn from a single urban area and was highly educated and White. Additional work should be done to understand the perspectives of other ethnicities, cultural groups, and regions that may experience different barriers and facilitators to exercise. In addition, the participants in this study may be more motivated to exercise than the average person with MCI or early dementia, as they volunteered to participate in research regarding exercise. As a result, their perceptions of exercise may also differ. Finally, social and behavioral phenomenon is thought to be contextually bound (Guba, 1981), meaning that perspectives and opinions of exercise are ever changing. This may be particularly true among persons living with MCI or early dementia who are likely to experience increasing needs for support as dementia progresses. Furthermore, changes in society's perspective on dementia are also likely to alter barriers and facilitators to exercise. Further longitudinal research is needed that follows persons with MCI or early dementia over their journey with dementia.

\section{Conclusion}

Our study revealed a complex and dynamic interplay between the individual, social network, and the community to influence exercise, where supports from the care partner, social network, and community can help persons living with MCI or early dementia to overcome individual-level barriers. Future research should continue to probe and develop interventions focused on the social and community level to enable exercise among persons living with MCI or early dementia. In addition, the influence of the social and physical environment should be further probed in diverse geographical and social communities, as this may be considered a more influential factor toward exercise among older adults with cognitive impairment due to societal perceptions and stigma. Understanding of the complex factors influencing exercise will contribute to guidelines and practical strategies to increase exercise among older adults with MCI or early dementia.

\section{Acknowledgments}

The authors would like to acknowledge the contributions of Matthew Vonk, Max Bergelt, and Cheyenne Mitchell to data collection as moderators and assistant moderators.

\section{References}

Alzheimer's Disease International and Dementia Alliance International. (2016, August). Access to CRPD and SDGs by persons with dementia. Retrieved from https://www.alz.co.uk/sites/default/files/ pdfs/access-crpd-dementia.pdf

Alzheimer's Europe. (2013). The ethical issues linked to the perceptions and portrayal of dementia and persons with dementia. Retrieved from https://www.alzheimer-europe.org/Ethics/Ethical-issues-in-practice/ 2013-The-ethical-issues-linked-to-the-perceptions-and-portrayal-ofdementia-and-people-with-dementia

Alzheimer's Society. (2019). Dementia-friendly sport and physical activity guide. Retrieved from https://www.alzheimers.org. uk/sites/default/files/2019-02/19003-Sports_Leisure_guide_online. pdf

Ashworth, R. (2017). Perceptions of stigma among persons affected by early- and late-onset Alzheimer's disease. Journal of Health Psychology, $1,1-21$.

Black, A.S., \& Rush, D.R. (2002). Cognitive and functional decline in adults aged 75 and older. Journal of the American Geriatrics Society, 50, 1978-1986. PubMed ID: 12473009 doi:10.1046/j.1532-5415. 2002.50609.x

Bechard, L.E., Mitchell, C.M., Regan, K., Bergelt, M.D., \& Middleton, L.E. (2018). Experiences, perceptions, and needs of community exercise providers concerning persons with mild cognitive impairment or dementia. Canadian Geriatrics Journal, 21, 71-133. doi:10.5770/ cgj.21.321

Boehm, J., Franklin, R.C., Newitt, R., Mcfarlane, K., Grant, T., \& Kurkowski, B. (2013). Barriers and motivators to exercise for older adults: A focus on those living in rural and remote areas of Australia. Australian Journal of Rural Health, 21(February), 141-149. doi:10. 1111/ajr.12032

Braun, V., \& Clarke, V. (2006). Using thematic analysis in psychology. Research in Psychology, 3, 77-101.

Cedervall, Y., \& Aberg, A.C. (2010). Physical activity and implications on well-being in mild Alzheimer's disease: A qualitative case study on two men with dementia and their spouses. Physiotherapy Theory and Practice, 26(August), 226-239. doi:10.3109/ 09593980903423012

Cedervall, Y., Torres, S., \& Aberg, A.C. (2015). Maintaining well-being and selfhood through physical activity: Experiences of persons with mild Alzheimer's disease. Aging \& Mental Health, 19(August), 679-688.

Chong, T.W.H., Doyle, C.J., Cyarto, E.V, Cox, K.L., Ellis, K.A., Ames, D., \& Lautenschlager, N.T. (2014). Physical activity program preferences and perspectives of older adults with and without cognitive impairment. Asia-Pacific Psychiatry: Official Journal of the Pacific Rim College of Psychiatrists, 6, 179-190. doi:10. 1111/appy.12015

Costello, E., Kafchinski, M., Vrazel, J., \& Sullivan, P. (2011). Motivators, barriers, and beliefs regarding physical activity in an older adult population. Journal of Geriatric Physical Therapy, 34(3), 138-147. PubMed ID: 21937904 doi:10.1519/JPT. 0b013e31820e0e71

Crowther, N. (2016, March 1). Harnessing the United Nations Convention on the Rights of Persons with Disabilities to improve the lives of persons with dementia. Retrieved from https://www.alz.co.uk/sites/ default/files/pdfs/harnessing-crpd-dementia.pdf

Dal Bello-Haas, V.P.M., Connell, M.E.O., Morgan, D.G., \& Crossley, M. (2014). Lessons learned: feasibility and acceptability of a telehealth-delivered exercise intervention for rural dwelling individuals with dementia and their caregivers. Rural and Remote Health, 14, 1-11.

David, R., Mulin, E., Friedman, L., Le Duff, F., Cygankiewicz, E., Deschaux, O., . . Z Zeitzer, J.M. (2012). Decreased daytime motor activity associated with apathy in Alzheimer disease: An actigraphic 
study. American Journal of Geriatric Psychiatry, 20, 806-814. PubMed ID: 21997602 doi:10.1097/JGP.0b013e31823038af

Forbes, C.S., Blake, M.C., Thiessen, J.E., \& Forbes, S. (2015). Exercise programs for persons with dementia. Cochrane Database of Systematic Reviews, 15(4), CD006489.

Franco, M.R., Tong, A., Howard, K., Sherrington, C., Ferriera, P.H., Pinto, R.Z., \& Ferreira, M.L. (2014). Older people's perspective on participation in physical activity: A systematic review and thematic analysis of qualitative literature. British Journal of Sports Medicine, 49, 12681276. doi:10.1136/bjsports-2014-094015

Ginis, K.A.M., Heisz, J., Spence, J.C., Clark, I.B., Antflick, J., Ardern, C.I., . . . Rotondi, M.A. (2017). Formulation of evidence-based messages to promote the use of physical activity to prevent and manage Alzheimer's disease. BMC Public Health, 17, 209. PubMed ID: 28212648 doi:10.1186/s12889-017-4090-5

Guba, E.G. (1981). Criteria for assessing the trustworthiness of naturalistic inquiries. Educational Communication and Technology Journal, 29(2), 75-91.

Henderson, D.X., \& Baffour, T. (2015). Applying a socio-ecological framework to thematic analysis using a statewide assessment of disproportionate minority contact in the United States. The Qualitative Report, 20(12), 1960-1973.

Husband, J.H. (2000). Diagnostic disclosure in dementia: an opportunity for intervention? Geriatric Psychiatry, 15(6), 544-547. PubMed ID: 10861922 doi:10.1002/1099-1166(200006)15:6<544::AID-GPS241 $>3.0 . \mathrm{CO} ; 2-8$

Kattari, S.K., Lavery, A., \& Hasche, L. (2017). Applying a social model of disability across the life span. Journal of Human Behavior in the Social Environment, 27(8), 865-880.

Krueger, R.A. (2000). Focus groups: A practical guide for applied research (3rd ed.). Thousand Oaks, CA: Sage.

Malthouse, R., \& Fox, F. (2015). Exploring experiences of physical activity among persons with Alzheimer's disease and their spouse carers: A qualitative study. Physiotherapy, 100, 169-175. PubMed ID: 24530168 doi:10.1016/j.physio.2013.10.002

Marcus, B.H., Selby, V.C., Niaura, R.S., \& Rossi, J.S. (1992). Selfefficacy and the stages of exercise behaviour change. Research Quarterly for Exercise and Sport, 63, 60-66. PubMed ID: 1574662 doi:10.1080/02701367.1992.10607557

Martins, J., Marques, A., Sarmento, H., \& Carreiro da Costa, F. (2015). Adolescents' perspectives on the barriers and facilitators of physical activity: A systematic review of qualitative studies. Health Education Research, 30, 742-755. PubMed ID: 26324394 doi:10.1093/her/ cyv042

McGuire, S. (2014). Centers for disease control and prevention. State Indicator on Physical activity, 2014. Atlanta, GA: U.S. Department of Health and Human Services; 2014. Advances in Nutrition, 5(6), 762763. PubMed ID: 25398737 doi:10.3945/an.114.007211

McLeroy, K.R., Bibeau, D., Steckler, A., \& Glanz, K. (1988). An ecological perspective on health promotion programs. Health Education Quarterly, 15, 351-377. PubMed ID: 3068205 doi:10.1177/ 109019818801500401

Menne, H.L., Kinney, J.M., \& Morhardt, D.J. (2002). Trying to continue to do as much as they can do: Theoretical insights regarding continuity and meaning making in the face of dementia. Dementia, 1(3), 367382. doi:10.1177/147130120200100308

Morgan, L.D. (1996). Focus groups as qualitative research: Planning and research design for focus groups. (3rd ed.). Thousand Oaks, CA: Sage.

O'Brien, M., Dodd, K.J., \& Bilney, B. (2008). A qualitative analysis of a progressive resistance exercise programme for persons with Parkinson's disease. Disability and Rehabilitation, 30, 1350-1357.
Öhman, H., Savikko, N., Strandberg, T.E., \& Pitkala, K.N. (2014). Effect of physical exercise on cognitive performance in older adults with mild cognitive impairment or dementia: A systematic review. Dementia and Geriatric Disorders, 38(5-6), 347-365.

Onwuegbuzie, A.J., Dickinson, W.B., Leech, N.L., \& Zoran, A.G. (2009). Toward more rigor in focus group research: A new framework for collecting and analyzing focus group data. International Journal of Qualitative Methods, 8, 1-21. Retrieved from http://ejournals.library. ualberta.ca/index.php/IJQM/article/view/4554

Onwuegbuzie, A.J., \& Leech, L.N. (2007). Validity and qualitative research: An oxymoron? Quality and Quantity, 41, 233-249. doi:10. 1007/s11135-006-9000-3

Petersen, R.C., Caracciolo, B., Brayne, C., Gauthier, S., Jelic, V., \& Fratiglioni, L. Mild cognitive impairment: A concept in evolution. Journal of Internal Medicine, 275(3), 214-228. doi:10.1111/joim. 12190

Phinney, A., Chaudhury, H., \& O'Connor, D.L. (2007). Doing as much as I can do: The meaning of activity for people with dementia. Aging and Mental Health, 11(4), 384-393.

Prince, M., Comas-Herrera, A., Knapp, M., Guerchet, M., \& Karagiannidou, M. (2016). World Alzheimer report 2016. London, UK: Alzheimer's Disease International.

Reed, P., \& Bluethmann, S. (2008). Voices of Alzheimer's disease: A summary report on the nationwide town hall meetings for persons with early stage dementia. Retrieved from https://www.alz.org/ media/Documents/early-stage-town-hall-r.pdf.

Schutzer, K.A., \& Graves, B.S. (2004). Barriers and motivations to exercise in older adults. Preventive Medicine, 39(2004), 1056-1061. doi:10. 1016/j.ypmed.2004.04.003

Shenton, K.A. (2014). Strategies for ensuring trustworthiness in qualitative research projects. Education for Information, 22, 63-75.

Strout, K.A., \& Howard, E.P. (2012). The six dimensions of wellness and cognition in older adults. Journal of Holistic Nursing, 30(3), 195-204. PubMed ID: 22713605 doi:10.1177/0898010112440883

Sutcliffe, C.L., Roe, B., Jasper, R., Jolley, D., \& Challis, D.J. (2015). Persons with dementia and carers' experiences of dementia care and services: Outcomes of a focus group study. Dementia, 14(6), 769787. PubMed ID: 24381211 doi:10.1177/1471301213511957

Suttanon, P., Hill, K.D., Said, C.M., Byrne, K.N., \& Dodd, K.J. (2012). Factors influencing commencement and adherence to a homebased balance exercise program for reducing risk of falls: perceptions of persons with Alzheimer's disease and their caregivers. International Psychogeriatrics/IPA, 24, 1172-1182. doi:10.1017/ S1041610211002729

Tak, E.C., van Uffelen, J.G., Paw, M.J., van Mechelen, W., \& HopmanRock, M. (2012). Adherence to exercise programs and determinants of maintenance in older adults with mild cognitive impairment. Journal of Aging and Physiology, 20, 32-46.

Thomas, C. (2004). How is disability understood? An examination of sociological approaches. Disability \& Society, 19(6), 569-583. doi:10.1080/0968759042000252506

United Nations. (2008, May). Convention on the Rights of Persons with Disabilities. Retrieved from https://treaties.un.org/doc/Publication/ CTC/Ch_IV_15.pdf

Van Alphen, H.J.M., Hortobágyi, T., \& van Heuvelen, M.J.G. (2016). Barriers, motivators, and facilitators of physical activity in dementia patients: A systematic review. Archives of Gerontology and Geriatrics, 66, 109-118. PubMed ID: 27295140 doi:10.1016/j.archger. 2016.05.008

World Health Organization. (2007). Global age-friendly cities: A guide. Retrieved from https://www.who.int/ageing/publications/age_friendly_ cities_guide/en/ 
Yu, F., \& Kolanowski, A. (2009). Facilitating aerobic exercise training in older adults with Alzheimer's Disease. Geriatric Nursing, 30(4), 250-259. PubMed ID: 19665667 doi:10.1016/j.gerinurse.2008. 11.001
Yu, F., Savik, K., Wyman, J.F., \& Bronas, U.G. (2011). Maintaining physical fitness and function in Alzheimer's disease: A pilot study. American Journal of Alzheimer's Disease and Other Dementias, 26, 406-412. doi:10.1177/1533317511414861 


\section{Focus Group Discussion Guides}

\section{Appendix A}

\section{Persons With $\mathrm{MCl}$ and Early Dementia}

1. What would you want people to know/understand about dementia?

2. What does exercise mean to you or how does exercise impact you (positively or negatively)?

a. Potential prompts:

i. For your health?

ii. For your brain/thinking ability?

iii. For your relationships and social functioning?

3. How has your condition (diagnosis) affected exercise opportunities?

4. What gets in the way of you doing exercise?

5 . What helps/motivates you to exercise?

6. What would the (your) ideal exercise opportunity look like?

a. Potential prompts:

i. Where would you like to exercise?

ii. Who would you like to exercise with?

iii. What would you like to do for exercise?

iv. If they would like group program: How long should each session be? How often should each session run? What costs should be associated with this program?

7. How would better exercise opportunities change your exercise habits/behaviors?

\section{Care Partners}

1. What would you want people to know/understand about dementia?

2. What does exercise mean to you or how does exercise impact you (positively or negatively)?

a. Potential prompts:

i. For your health?

ii. For your brain/thinking ability?

iii. For your relationships and social functioning?

iv. Exercise history

3. How has your partner's condition (diagnosis) affected exercise opportunities?

4. What gets in the way of you and your partner doing exercise?

5 . What helps/motivates you and your partner to exercise?

6. What would the (your) ideal exercise opportunity look like?

a. Potential prompts:

i. Where would you like to exercise?

ii. Who would you like to exercise with?

iii. What would you like to do for exercise?

iv. (If they would like group program: How long should each session be? How often should each session run? What costs should be associated with this program?

7. How would better exercise opportunities change your exercise habits/behaviors? 